\title{
Zur Stärkung der Solidarität bei der Pflegefinanzierung
}

Stefan Greß, Dietmar Haun und Klaus Jacobs

19.1 Einleitung - 242

19.2 Finanzierungsoption Bundeszuschuss? - 242

19.3 Stärkung der Solidarität innerhalb der Pflegeversicherung - 245

19.3.1 Gravierende Strukturunterschiede zwischen den beiden Zweigen der gesetzlichen Pflegeversicherung - 245

19.3.2 Auswirkungen systemübergreifender Solidarität - 250

$19.4 \quad$ Fazit -252

Literatur - 253 


\section{- - Zusammenfassung}

Nach wiederholten Anhebungen des Beitragssatzes zur sozialen Pflegeversicherung in kurzer Zeit steht die künftige Ausgestaltung der Pflegefinanzierung weiterhin auf der politischen Agenda. Zwei Anknüpfungspunkte für Finanzierungsreformen der Pflegeversicherung sind Gegenstand dieses Beitrags: die Einführung eines steuerfinanzierten Zuschusses zur sozialen Pflegeversicherung nach dem Vorbild der gesetzlichen Krankenversicherung sowie die Einbeziehung aller gesetzlich Pflegeversicherten, also auch der Versicherten der privaten Pflegepflichtversicherung, in die solidarische Finanzierung. Während die Beurteilung eines Bundeszuschusses im Unterschied zu vielen aktuellen Forderungen eher zurückhaltend ausfällt, wird die Einbeziehung aller Versicherten in die solidarische Finanzierung angesichts der gravierenden Strukturunterschiede zwischen sozialer Pflegeversicherung und privater Pflegepflichtversicherung als ein längst überfälliger Schritt angesehen. Unter Statusquo-Bedingungen der Beitragsgestaltung ergäbe sich hieraus ein Entlastungseffekt für die soziale Pflegeversicherung von rund 0,4 Beitragssatzpunkten.

After the contribution rate to social long-term care insurance has been raised repeatedly in a short time, the future structure of long-term care financing remains on the political agenda. The paper deals with two starting points for financing reforms in this area: the introduction of a tax-financed subsidy for social long-term care insurance along the lines of statutory health insurance and the inclusion of all persons insured under the statutory long-term care insurance system, including those insured with private compulsory long-term care insurance, in solidaritybased financing. While, in contrast to many current demands, the assessment of a federal subsidy is rather cautious, the inclusion of all insurees in solidarity-based financing is regarded as a long overdue step in view of the serious structural differences between social long-term care insurance and private compulsory long-term care insurance. In the present system of calculation, this would reduce the contribution rate of social long- term care insurance by about 0.4 percentage points.

\subsection{Einleitung}

Der Beitragssatz zur sozialen Pflegeversicherung (SPV) ist zum Jahresbeginn 2019 um 0,5 Prozentpunkte angehoben worden. Dies war bereits die dritte Erhöhung seit 2015 um insgesamt einen vollen Prozentpunkt. Zwar wird erwartet, dass der aktuelle Beitragssatz von 3,05\% bzw. 3,3\% für Kinderlose ausreichen dürfte, um die Ausgaben der SPV bis einschließlich 2022 zu decken. Dies gilt aber schon nicht mehr für weitere von der Bundesregierung angestrebte Maßnahmen, mit denen die Personalausstattung in der professionellen Pflege verbessert und höhere Gehälter in der Langzeitpflege gezahlt werden sollen, um die Attraktivität der Pflegeberufe zu erhöhen (Schwinger et al. 2018). Eine grundlegende Debatte zur weiteren Ausgestaltung der Finanzierung des Pflegerisikos ist somit unumgänglich.

In diesem Beitrag sollen zwei mögliche Anknüpfungspunkte dieser Debatte näher beleuchtet werden: die aktuell wiederholt geforderte Einführung eines steuerfinanzierten Bundeszuschusses zur SPV und die Auswirkungen der derzeit vergleichsweise wenig diskutierten Dualität innerhalb der vor einem Vierteljahrhundert eingeführten gesetzlichen Pflegeversicherung mit der SPV auf der einen und der privaten Pflegepflichtversicherung (PPV) auf der anderen Seite.

\subsection{Finanzierungsoption Bundeszuschuss?}

Vor dem Hintergrund weiterhin steigender Ausgaben in der SPV werden zunehmend Forderungen nach einem Bundeszuschuss erhoben. So hat sich die 95. Arbeits- und Sozialministerkonferenz der Bundesländer (ASMK) am 5. Dezember 2018 in Münster mehrheitlich für einen solchen Steuerzuschuss ausgesprochen. 
In dem Beschluss wird die Bundesregierung gebeten, „den Wert der Leistungen, die die Pflegeversicherung vordringlich im gesamtgesellschaftlichen Interesse erbringt sowie die Höhe entsprechend entgehender Einnahmen zu ermitteln und auf dieser Basis einen finanziellen Zuschuss aus dem Bundeshaushalt an den Ausgleichsfonds der sozialen Pflegeversicherung zu etablieren" (ASMK 2018, S. 15). Im Frühjahr 2019 haben die Länder Hamburg, Berlin, Bremen und Schleswig-Holstein im Bundesrat einen Entschließungsantrag zur Weiterentwicklung der Pflegeversicherung eingebracht. Dieser sieht als eine von drei geforderten Reformmaßnahmen - neben der Übernahme der Kosten der medizinischen Behandlungspflege in Pflegeheimen durch die gesetzliche Krankenversicherung (GKV) und einer gesetzlichen Begrenzung der Eigenanteile der Pflegebedürftigen an den Pflegekosten - einen dynamischen Zuschuss aus dem Bundeshaushalt an den Ausgleichsfonds der sozialen Pflegeversicherung vor. Dessen Höhe soll sich in einem ersten Schritt „am Wert der Leistungen (orientieren), die die Pflegeversicherung derzeit vordringlich im gesamtgesellschaftlichen Interesse erbringt" (Bundesrat 2019, S. 1).

Auch der GKV-Spitzenverband spricht sich für einen Bundeszuschuss zur SPV als „systemkonforme Finanzierungsmöglichkeit“ aus, die „Zu einer ausgewogeneren Finanzierung der gesamtgesellschaftlichen Aufgabe Pflege beitragen und die Beitragszahlerinnen und Beitragszahler entlasten (könnte)“; er beziffert das Volumen der Ausgaben für versicherungsfremde Leistungen im Jahr 2018 auf mindestens 2,7 Mrd. $€$ (GKV-Spitzenverband 2018a). Demgegenüber hat Bundesgesundheitsminister Spahn Forderungen nach einem Bundeszuschuss bisher abgelehnt: Das Problem der versicherungsfremden Leistungen sehe er in der Pflegeversicherung ,in diesem Maße“ nicht. ${ }^{1}$

Forderungen nach einem Bundeszuschuss zur SPV orientieren sich zumeist an dem im Jahr 2004 eingeführten Bundeszuschuss für die

Vgl. Interview im Tagesspiegel vom 16.10.2018 (Spahn 2018).
GKV, mit dem sich der Bund gemäß $\$ 221$ SGB V „zur pauschalen Abgeltung der Aufwendungen der Krankenkassen für versicherungsfremde Leistungen“" an der GKV-Finanzierung beteiligt. Allerdings ist der Begriff der versicherungsfremden Leistungen nicht unumstritten, weil zu diesen Leistungen vor allem die beitragsfreie Mitversicherung von Kindern und Jugendlichen sowie von Ehegatten gezählt wird, die durchaus auch als Kernaufgabe einer Sozialversicherung betrachtet werden kann. Insofern wäre es sachgerechter, von gesamtgesellschaftlichen Aufgaben zu sprechen. Doch sind sowohl der Begriff der versicherungsfremden Leistungen als auch der der gesamtgesellschaftlichen Aufgaben unpräzise, weil es keinen Konsens über das Ausmaß dieses Leistungs- bzw. Aufgabenspektrums gibt. Demzufolge gibt es auch keine klare Regelbindung für die Höhe des Bundeszuschusses nach $\$ 221$ SGB V. $^{2}$ Als Konsequenz wurde die Höhe dieses Bundeszuschusses seit seiner Einführung im Jahr 2004 mehrfach erhöht und wieder abgesenkt (Greß und Bieback 2014). Derzeit finanziert der Bund 14,5 Mrd. € pro Jahr. Dieser Betrag liegt deutlich unter den Aufwendungen für die oben angesprochene beitragsfreie Mitversicherung, die der GKV-Spitzenverband für das Jahr 2011 auf 29,8 Mrd. € geschätzt hat (GKVSpitzenverband 2013).

Vor diesem Hintergrund stellt sich die Frage, wie ein Bundeszuschuss zur SPV gerechtfertigt werden könnte. Dabei zeigt sich, dass die Analogie zur GKV rasch an Grenzen stößt, denn die beitragsfreie Mitversicherung insbesondere von Kindern und Jugendlichen kommt als Rechtfertigungsgrund keineswegs eindeutig in Betracht. Das Bundesverfassungsgericht hat in seinem wegweisenden Urteil aus dem Jahr 2001 hierzu eine außerordentlich konsistente Argumentationskette aufgebaut. Das Gericht beurteilte die bis dahin geltende Beitragsfinanzierung als verfassungswidrig, weil die Betreuung und Erziehung von Kindern bei der

2 Eine Zweckbindung von Steuermitteln ist im Gegensatz zur Beitragsfinanzierung ohnehin nicht möglich. 
Beitragsbemessung nicht berücksichtigt worden sei. Damit wäre die Gruppe der Versicherten mit Kindern gegenüber kinderlosen Versicherten in verfassungswidriger Art und Weise benachteiligt. Schließlich würden letztere aus der Betreuungs- und Erziehungsleistung ersterer im Falle ihrer Pflegebedürftigkeit einen Nutzen ziehen (BVerfG 2001a, Rn. 55).

Zwar würden Kinderlose mit ihren Beiträgen auch zur Finanzierung des Pflegerisikos der beitragsfrei Mitversicherten herangezogen. Das würde jedoch den Vorteil der kinderlosen Versicherten zu Lasten derjenigen nicht aufwiegen, die zur Abdeckung des Pflegerisikos aller im Alter für die zukünftigen Beitragszahler sorgen würden (BVerfG 2001a: Rn. 58). Das Gericht zog daher die Schlussfolgerung: „Die gleiche Belastung mit Versicherungsbeiträgen führt zu einem erkennbaren Ungleichgewicht zwischen dem Gesamtbeitrag, den Kindererziehende in die Versicherung einbringen, und dem Geldbeitrag von Kinderlosen. Hierin liegt eine Benachteiligung von erziehenden Versicherten, die im Beitragsrecht auszugleichen ist" (BVerfG 2001a, Rn. 67; Hervorhebung durch die Verf.). Dem ist der Gesetzgeber nachgekommen, indem Kinderlose seit dem 1. Januar 2005 einen um 0,25 Prozentpunkte erhöhten einkommensabhängigen Beitragssatz zur SPV aufbringen müssen, an dem der Arbeitgeber nicht beteiligt ist.

Auch die Argumentation im Hinblick auf gesamtgesellschaftliche Aufgaben der SPV in Gestalt von Leistungen zur sozialen Absicherung von nicht professionell tätigen Pflegepersonen ( $\$ 44$ SGB XI) ist kein Selbstläufer. Es ist zwar korrekt, dass die Pflegeversicherung mit der sozialen Absicherung dieser Personen aus Sicht der SPV an sich sachfremde Aufgaben finanziert, weil es sich nicht unmittelbar um Pflegeleistungen handelt. Es lässt sich aber ebenso gut argumentieren, dass die ambulante Laienpflege grundsätzlich günstiger für die Beitragszahler ist als professionell organisierte Pflege und insofern die soziale Absicherung von Pflegepersonen die Attraktivität von Laienpflege und damit auch das Potenzial zur Begrenzung der Ausgaben erhöht.
Die Rechtfertigung eines Bundeszuschusses mit der Kompensation der Pflegeversicherung für die Wahrnehmung gesamtgesellschaftlicher Aufgaben steht damit insgesamt auf einer eher schmalen argumentativen Basis. Das gilt auch für die Aussage in dem bereits zitierten Entschließungsantrag von Hamburg und drei weiteren Ländern, dass die Solidarität - wie in anderen Zweigen der Sozialversicherung - durch einen dynamisierten Zuschuss aus dem Bundeshaushalt auf eine noch breitere Basis gestellt werde (Bundesrat 2019, S. 5). Unbestritten weist die derzeitige Beitragsfinanzierung in der gesetzlichen Kranken- und der sozialen Pflegeversicherung im Vergleich zur Steuerfinanzierung erhebliche Defizite im Hinblick auf horizontale und vertikale Gerechtigkeitsprinzipien auf - angefangen damit, dass Privatversicherte überhaupt nicht an der solidarischen Finanzierung beteiligt sind (Greß und Bieback 2014). Diese Defizite ließen sich aber sukzessive beseitigen, zumal eine Verlagerung der Einkommensumverteilungsfunktion von der GKV in das Steuersystem seit dem Scheitern des Modells der Kopfpauschale bzw. Gesundheitsprämie aus guten Gründen nicht mehr auf der politischen Tagesordnung steht.

Letztlich sind die Erfahrungen mit der Finanzierungsoption Steuerfinanzierung aber auch im Kontext der SPV nicht uneingeschränkt positiv. Mit der Einführung der SPV sollten die Bundesländer bei der Sozialhilfe auf der einen Seite finanziell entlastet werden, um auf der anderen Seite die Pflegebedürftigen bei den Investitionskosten unterstützen zu können ( $\$ 9$ SGB XI). Mit Ausnahme eines vom Bund finanzierten Investitionsprogramms in den ostdeutschen Bundesländern sind die Bundesländer dieser Verantwortung jedoch nur unzureichend nachgekommen (Rothgang 2018). Als Konsequenz steigen die Eigenanteile der Pflegebedürftigen zur Finanzierung der nicht öffentlich finanzierten Investitionskosten kontinuierlich. Der bereits zitierte Entschließungsantrag lässt zudem nicht erkennen, dass die Länder in diesem Zusammenhang für sich selbst überhaupt eine Finanzierungsaufgabe sehen. Dass Pflegebedürftige 
die Investitionskosten „im Sinne von Kaltmiete“ selbst tragen müssten, sei schließlich „breit akzeptiert" (Bundesrat 2019, S. 3).

Zusammenfassend erscheint die Finanzierungsoption Bundeszuschuss für die SPV keineswegs derart vielversprechend, wie derzeit von verschiedenen Seiten der Eindruck erweckt wird. Erstens dürfte das Finanzierungsvolumen der von der SPV erfüllten gesamtgesellschaftlichen Aufgaben im Vergleich zur GKV vergleichsweise gering ausfallen. Zweitens ist eine Beseitigung der Gerechtigkeitsdefizite in der SPV einem Auf- bzw. Ausbau der Steuerfinanzierung vorzuziehen. Dies gilt vor allem, weil drittens die Erfahrungen mit der Finanzierungsoption Steuerfinanzierung nicht nur in anderen Sozialversicherungszweigen keineswegs uneingeschränkt positiv ausfallen, sondern angesichts der mangelnden öffentlichen Finanzierung von Investitionskosten auch bereits in der SPV.

\subsection{Stärkung der Solidarität innerhalb der Pflegeversicherung}

Während die Forderung nach einem Bundeszuschuss zur SPV zur Flankierung der Beitragsfinanzierung in der SPV derzeit augenscheinlich Konjunktur hat, ist es im Hinblick auf eine andere Reformoption zur Stärkung der Solidarität bei der Pflegefinanzierung vergleichsweise still. Die Rede ist von einer Beteiligung aller Versicherten an der solidarischen Finanzierung der gesamten gesetzlichen Pflegeversicherung, die aus den beiden Zweigen der SPV und der PPV besteht. Als das Bundeskabinett im Herbst 2018 den Entwurf für das zwischenzeitlich zum Jahresbeginn 2019 in Kraft getretene Gesetz zur Anhebung des SPV-Beitrags um 0,5 Prozentpunkte beschloss, trug eine Pressemitteilung des Bundesgesundheitsministeriums die Überschrift: „Bundesgesundheitsminister Jens Spahn: Gute Pflege muss uns als Solidargemeinschaft etwas wert sein" (BMG 2018a). Wen meint Jens Spahn eigentlich mit „uns“? Er selbst ist wie über neun Millionen weitere Personen privat pflegeversichert und somit überhaupt nicht Teil der Solidargemeinschaft, auf die sich seine Aussage ausschließlich bezieht.

In Anlehnung an das duale Krankenversicherungssystem hatte der Gesetzgeber 1994 eine zweigeteilte Pflegepflichtversicherung eingeführt, bestehend aus der SPV als einer auf einkommensbezogenen Beiträgen basierenden Sozialversicherung nach dem Umlageverfahren und der in der Praxis überwiegend durch altersabhängige Prämien finanzierten PPV, für die das Kapitaldeckungssystem gilt. Wie das Bundesverfassungsgericht 2001 in seinem Urteil zur Verfassungsmäßigkeit der PPV ausführte, durfte „der Gesetzgeber, der eine Pflegevolksversicherung in der Gestalt zweier Versicherungszweige geschaffen hat, (...) die einzelnen Gruppen dem einen oder anderen Versicherungszweig sachgerecht und unter dem Gesichtspunkt einer ausgewogenen Lastenverteilung zuordnen“ (BVerfG 2001b, Rn. 92). Inwiefern die Lastenverteilung zwischen sozialer und privater Pflegeversicherung tatsächlich ausgewogen ist, war nicht Gegenstand der richterlichen Prüfung. Allerdings lässt sich diese Frage empirisch untersuchen, was nachfolgend in Bezug auf zentrale Strukturmerkmale von SPV- und PPV-Versicherten geschieht.

\subsubsection{Gravierende Strukturunterschiede zwischen den beiden Zweigen der gesetzlichen Pflegeversicherung}

Die Grundlage der folgenden Analyse bilden die Mikrodaten des sozio-oekonomischen $\mathrm{Pa}-$ nels (SOEP), einer seit dem Jahr 1984 jährlich durchgeführten repräsentativen Wiederholungsbefragung von Personen in privaten Haushalten in Deutschland (Goebel et al. 2018). Persönlich befragt werden im SOEP in 
den ausgewählten Panelhaushalten sämtliche Personen im Alter von 17 Jahren und älter. Entsprechend bildet die erwachsene Wohnbevölkerung in Deutschland die Analysepopulation. ${ }^{3}$ Die Zugehörigkeit zur SPV bzw. PPV beruht auf der Frage zur Art der Krankenversicherung. Aufgrund der gesetzlichen Regelung, dass die Zuweisung zur Pflegeversicherung der Zugehörigkeit zur Krankenkasse (GKV oder PKV) folgt, kann der Status der Personen bis auf wenige Ausnahmen valide bestimmt werden.

Das Risiko der Pflegebedürftigkeit ist in hohem Maße vom Lebensalter abhängig und weist bei Älteren zudem deutliche Unterschiede zwischen Männern und Frauen auf. Ein direkter Vergleich der altersspezifischen Pflegerisiken zwischen SPV und PPV-Versicherten ist nicht möglich, da für die PPV keine diesbezüglichen Daten vorliegen. Daher werden die durch das Statistische Bundesamt für die deutsche Bevölkerung insgesamt ermittelten Pflegerisiken nach Altersgruppen und Geschlecht dargestellt. So weist das Statistische Bundesamt für das Jahr 2017 für alle Personen unter 60 Jahren eine Pflegequote von unter $0,9 \%$ aus. In den Altersjahrgängen ab 60 Jahren nimmt der Anteil der Pflegebedürftigen an der Altersgruppe insgesamt von $2 \%$ bis zum Alter von 79 Jahren auf rund $11 \% \mathrm{zu}$. Unterschiede in den Pflegequoten zwischen Männern und Frauen sind bis zum 75. Lebensjahr kaum festzustellen. Vom 80. Lebensjahr an nimmt das Risiko von Pflegebedürftigkeit jedoch stark zu: Mehr als jeder Dritte im Alter von über 80 Jahren nahm 2017 Pflegeleistungen in Anspruch. Von den 3,4 Mio. Pflegebedürftigen im Jahr 2017 hatten $55 \%$ das 80. Lebensjahr überschritten. In Verbindung mit der höheren Lebenserwartung waren von

3 Im Auftrag des Deutschen Instituts für Wirtschaftsforschung (DIW) werden im Erhebungsprogramm des SOEP jedes Jahr in Deutschland über 25.000 Personen aus rund 16.000 Haushalten von Kantar Public befragt. Den hier dargestellten SOEP-Analysen für das Jahr 2016 liegt eine Nettostichprobe von 24.559 realisierten Befragungen von Personen im Alter von 18 Jahren und älter zugrunde. den 1,9 Mio. Pflegebedürftigen über 80 Jahren $72 \%$ Frauen (Statistisches Bundesamt 2018).

Vor diesem Hintergrund werden mit Hilfe der Analyse von SOEP-Daten verschiedener Erhebungswellen die Alters- und Geschlechtsstrukturen der SPV- und PPV-Versicherten in den Jahren 1995, 2005 und 2016 verglichen. Danach waren in der SPV im Vergleich zur PPV zur Zeit der Einführung der Pflegeversicherung Mitte der 1990er-Jahre die mittleren Altersgruppen in der PPV überproportional vertreten (- Abb. 19.1). ${ }^{4}$ Altersjahrgänge mit hoher Pflegeprävalenz waren dagegen deutlich seltener privat pflegeversichert. So war speziell der Anteil der über 80-Jährigen in der PPV nur halb so groß wie in der SPV. An diesen strukturellen Unterschieden hat sich auch zehn Jahre später wenig geändert. Erst 2016 ist partiell eine Angleichung der Altersstruktur zwischen PPV und SPV festzustellen: Der Anteil der 60- bis 79-jährigen Privatversicherten liegt mit 34,7 \% jetzt sogar über dem Anteil in der SPV. Bei der Altersgruppe mit der höchsten Pflegequote, den über 80-Jährigen, liegt der Versichertenanteil in der SPV mit 6,4\% jedoch weiterhin um fast die Hälfte über dem entsprechenden Anteil in der PPV. Hinzu kommt, dass in der PPV nur unterdurchschnittlich häufig Frauen versichert sind. 2016 lag der Frauenanteil in der PPV bei $39 \%$, in der SPV hingegen bei $53 \%$. In Verbindung mit ihrer höheren Lebenserwartung tragen Frauen ein signifikant höheres Pflegerisiko als Männer. ${ }^{5}$

Auch Kinder und Jugendliche sowie Personen im erwerbsfähigen Alter (bis 64 Jahren)

4 Diese und nachfolgende Ergebnisse des SOEP werden personengewichtet und zugleich auf den Querschnitt der Bevölkerung im Erhebungsjahr hochgerechnet ausgewiesen.

5 Letztlich ist das Schadensrisiko der PPV noch ein ganzes Stück geringer, weil knapp die Hälfte ihrer Versicherten 2016 einen staatlichen Beihilfeanspruch hatten. Unter den beihilfeberechtigten Versicherten über 18 Jahren liegt der Frauenanteil bei $50 \%$, bei den nicht-beihilfeberechtigten jedoch nur bei $27 \%$ (PKV 2017). Für den Personenkreis der beihilfeberechtigten PPV-Versicherten übernimmt die steuerfinanzierte Beihilfe in der Regel mindestens $50 \%$ der Ausgaben für Pflegeleistungen. 


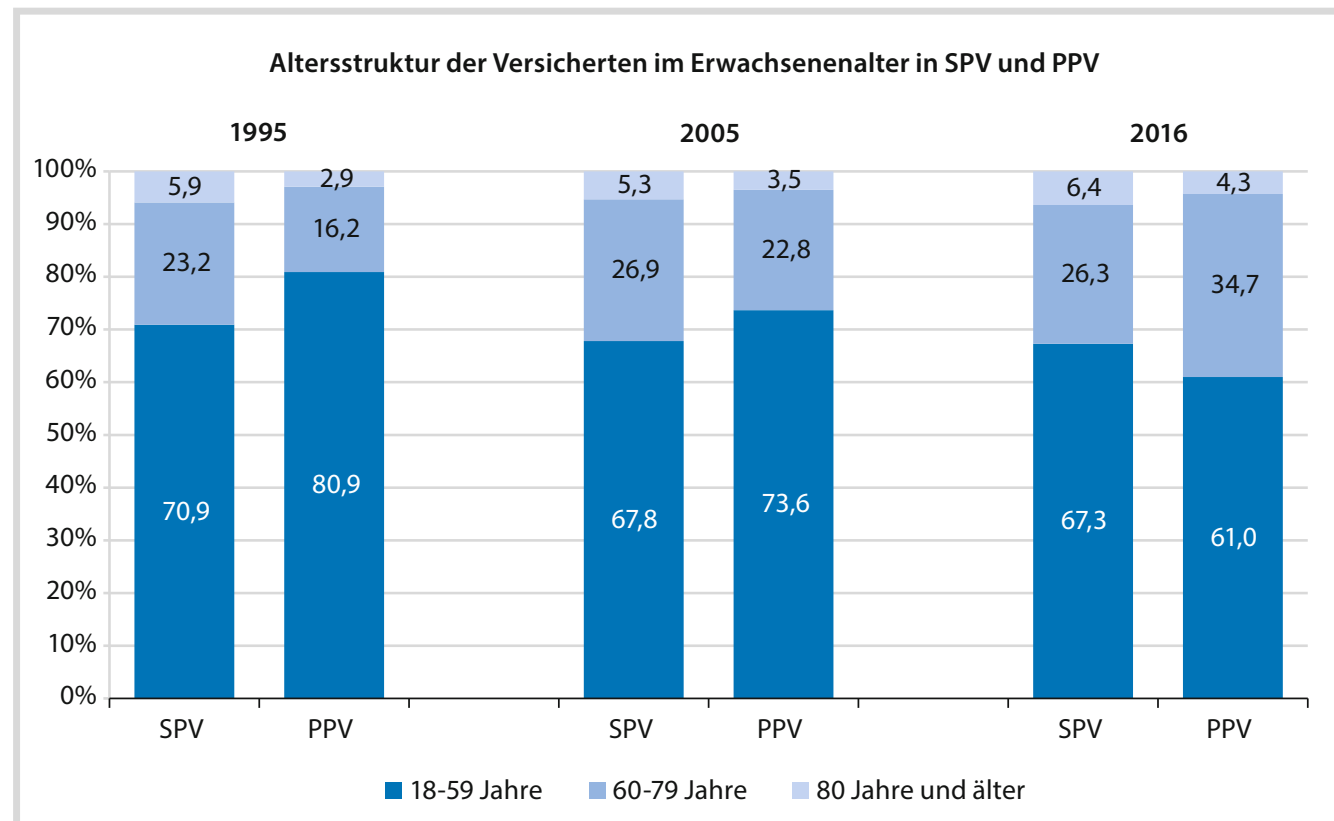

Pflege-Report 2019

- Abb. 19.1 Altersstruktur der Versicherten im Erwachsenenalter in SPV und PPV 1995, 2005 und 2016. (Quelle: Eigene Berechnungen auf Basis des SOEP, Population: Personen in Privathaushalten 18 Jahre und älter)

sind - wenn auch in geringerem Ausmaß vom Risiko der Pflegebedürftigkeit betroffen. Der Anteil dieser Altersgruppen an den Pflegebedürftigen lag 2017 bundesweit bei rund 19\% (Statistisches Bundesamt 2018, S. 19). Allerdings vermittelt die Alters- und Geschlechtsstruktur der SPV- und PPV-Versicherten nur einen ersten Eindruck von den beträchtlichen Unterschieden im Pflegerisiko der beiden Versicherungszweige, weil weitere Ursachen für systematische Risikounterschiede außerhalb der Betrachtung bleiben. So besteht insbesondere für Personen mit Vorerkrankungen und Behinderungen beim Zugang zur PKV aufgrund der dort üblichen Gesundheitsprüfung sowie fehlenden Kontrahierungszwangs eine vielfach unüberwindliche Hürde. Somit gibt es systembedingt gerade in den jüngeren und mittleren Altersgruppen ein deutlich geringeres altersspezifisches Pflegerisiko in der PPV, das im Altersbereich zwischen 20 und 50 Jahren teilweise nicht einmal $20 \%$ des ent- sprechenden Werts der SPV-Versicherten beträgt, weil sich die Selektionswirkung der Risikoprüfung hier besonders stark bemerkbar macht (DAV 2015, S. 12). ${ }^{6}$

Gravierende Strukturunterschiede zwischen SPV und PPV bestehen auch im Hinblick auf die ökonomische Leistungsfähigkeit der Versicherten. Nach den Analyseergebnissen des SOEP waren 2016 über 60 Mio. Menschen

6 Die Expertise der Deutschen Aktuarvereinigung e. V. ist eine der wenigen öffentlich zugänglichen Studien, in der die alters- und geschlechtsspezifischen Pflegeprävalenzen der SPV mit Hilfe von Daten des PKV-Verbandes für das Jahr 2004 direkt mit denen der PPV vergleichen werden. Die geringeren Pflegeprävalenzen in der PPV bei den über 50-Jährigen fassen die Aktuare folgendermaßen zusammen: „In höheren Altern bestehen allerdings immer noch erhebliche Unterschiede zwischen privater und sozialer Pflegepflichtversicherung, die nicht allein mit der oft Jahrzehnte zurückliegenden Risikoprüfung erklärt werden können. Vielmehr scheinen auch sozioökonomische Effekte vorzuliegen" (DAV 2015, S. 12). 
Tabelle 19.1 Stellung im Erwerbssystem und jährliches Durchschnittseinkommen von SPV- und PPVVersicherten 2016

\begin{tabular}{|c|c|c|c|c|c|}
\hline & \multicolumn{3}{|c|}{ Anzahl und Anteil an Versicherten } & \multicolumn{2}{|c|}{$\begin{array}{l}\text { Bruttogesamteinkommen } \\
\text { je Versicherten, in Euro }\end{array}$} \\
\hline & SPV & PPV & Gesamt & SPV & PPV \\
\hline \multirow{2}{*}{$\begin{array}{l}\text { Arbeiter, einfache und } \\
\text { mittlere Angestellte }\end{array}$} & 25.688 .167 & 454.819 & 26.142 .986 & & \\
\hline & $42,4 \%$ & $5,6 \%$ & $38,0 \%$ & 28.050 & 38.167 \\
\hline \multirow{2}{*}{$\begin{array}{l}\text { Hochqualifizierte und } \\
\text { leitende Angestellte }\end{array}$} & 5.436 .529 & 947.175 & 6.383 .704 & & \\
\hline & $9,0 \%$ & $11,7 \%$ & $9,3 \%$ & 53.348 & 102.448 \\
\hline \multirow[t]{2}{*}{ Beamte } & 301.910 & 2.222 .613 & 2.524 .523 & & \\
\hline & $0,5 \%$ & $27,5 \%$ & $3,7 \%$ & 47.248 & 46.953 \\
\hline \multirow{2}{*}{$\begin{array}{l}\text { Selbständige, Freie } \\
\text { Berufe (mit Mitarbeitern) }\end{array}$} & 629.512 & 698.035 & 1.327.547 & & \\
\hline & $1,0 \%$ & $8,6 \%$ & $1,9 \%$ & 58.572 & 91.281 \\
\hline \multirow{2}{*}{$\begin{array}{l}\text { Selbständige, Freie } \\
\text { Berufe (ohne Mitarbeiter) }\end{array}$} & 1.782 .380 & 653.103 & 2.435 .483 & & \\
\hline & $2,9 \%$ & $8,1 \%$ & $3,5 \%$ & 30.157 & 75.621 \\
\hline \multirow[t]{2}{*}{ Rentner, Pensionär } & 16.021 .718 & 2.400 .941 & 18.422.659 & & \\
\hline & $26,4 \%$ & $29,7 \%$ & $26,8 \%$ & 17.660 & 34.825 \\
\hline \multirow{2}{*}{$\begin{array}{l}\text { Studierende, } \\
\text { Auszubildende }\end{array}$} & 4.198.727 & 370.777 & 4.569 .504 & & \\
\hline & $6,9 \%$ & $4,6 \%$ & $6,6 \%$ & 7.849 & 5.247 \\
\hline \multirow{2}{*}{$\begin{array}{l}\text { Arbeitslose und sonstige } \\
\text { Nichterwerbstätige }\end{array}$} & 6.572 .413 & 337.442 & 6.909 .855 & & \\
\hline & $10,8 \%$ & $4,2 \%$ & $10,1 \%$ & 10.903 & 15.757 \\
\hline \multirow[t]{2}{*}{ Gesamt } & 60.631 .356 & 8.084 .905 & 68.716.261 & 24.790 & 52.287 \\
\hline & 88,2 & 11,8 & 100,0 & & \\
\hline
\end{tabular}

bzw. $88,2 \%$ der Bevölkerung im Erwachsenenalter SPV- und 11,8\% PPV-versichert (- Tab. 19.1). Als Folge der selektiven Zugangsmodalitäten sind die PPV-Versicherten $\mathrm{zu}$ einem deutlich höheren Anteil voll erwerbstätig und nehmen gehobene Positionen im Erwerbssystem ein. Neben Beamten und Pensionären sind hochqualifizierte und leitende Angestellte sowie Selbständige und Freiberufler überproportional häufig privat pflegeversichert. Arbeiter und einfache An- gestellte wie auch Arbeitslose und sonstige Nichterwerbstätige sind demgegenüber in der PPV unterrepräsentiert. ${ }^{7}$ Somit ist wenig verwunderlich, dass die PPV-Versicherten über ein Brutto-Gesamteinkommen verfügen, das im Durchschnitt mit 52.287€ pro Jahr mehr als doppelt so hoch ist wie das mittlere Jahresein-

Zu Unterschieden der Erwerbs- und Einkommensstrukturen in der Krankenversicherung zwischen Versicherten der GKV und der PKV siehe Haun 2013. 
- Tabelle 19.2 Finanzkennzahlen zu SPV und PPV 2005 und 2017

\begin{tabular}{|c|c|c|c|c|}
\hline & SPV & PPV & SPV & PPV \\
\hline & \multicolumn{2}{|l|}{2005} & \multicolumn{2}{|l|}{2017} \\
\hline Anzahl Versicherte (in Tsd.) & 70.522 & 9.164 & 72.240 & 9.327 \\
\hline Beitragseinnahmen in Mrd. Euro & 17,38 & 1,87 & 36,10 & 2,59 \\
\hline Beitrag/Prämie je Versicherten und Jahr in Euro & 246 & 204 & 500 & 278 \\
\hline Anzahl Pflegebedürftige (in Tsd.) & 1.952 & 116 & 3.302 & 212 \\
\hline Anzahl Pflegebedürftige je 100 Versicherte & 2,77 & 1,27 & 4,57 & 2,27 \\
\hline Leistungsausgaben in Mrd. Euro & 16,98 & 0,55 & 35,54 & 1,23 \\
\hline Leistungsausgaben je Versicherten in Euro & 241 & 60 & 492 & 131 \\
\hline Leistungsausgaben je Vers. in Euro inklusive Beihilfeausgaben & 241 & 90 & 492 & 197 \\
\hline Leistungsausgaben je Pflegebedürftigen in Euro & 8.699 & 4.744 & 10.763 & 5.790 \\
\hline Mittelbestand/Altersrückstellungen in Mrd. Euro & 3,05 & 15,17 & 6,9 & 34,5 \\
\hline
\end{tabular}

kommen der SPV-Versicherten, das $24.790 €$ beträgt.

Die Auswirkungen der gezeigten Strukturunterschiede beim primär alters- und geschlechtsbedingten Pflegerisiko sowie beim Einkommen der SPV- und PPV-Versicherten finden ihren Niederschlag in den in - Tab. 19.2 dargestellten Kennzahlen zu den beiden Versicherungszweigen, auch in ihrer zeitlichen Entwicklung. ${ }^{8}$ Bereits 2005 überstieg die versichertenbezogene Durchschnittsprämie der PPV von $204 €$ ihre mittleren Leistungsausgaben von $60 €$ je Versicherten bei Weitem. Der überwiegende Teil der Beitragseinnahmen floss in den Aufbau der Alterungsrückstellungen: Dies waren 2005 nach Angaben des PKV-Verbands sogar 93,3\% der Beitragseinnahmen (PKV 2006). Zur Finanzierung der Leistungsausgaben von rd. 0,5 Mrd. Euro dienten wohl vor allem Kapitalerträge und Überschüsse aus den Vorjahren. Jedenfalls wurden die Beitragseinnahmen

8 Die konzeptionelle Vorlage für •Tab. 19.2 liefern Dräther et al. 2009 mit ihrer Tabelle 3.1. der PPV hierfür nur zu einem Bruchteil beansprucht.

Ganz anders stellt sich die Finanzsituation in der SPV dar, in der gegenüber der PPV im Jahr 2005 über 70 Mio. Menschen mit einem mehr als doppelt so hohen Anteil an Pflegebedürftigen versichert waren. Die Leistungsausgaben beliefen sich mit durchschnittlich $241 €$ je Versicherten sogar auf den vierfachen Betrag. Zur Finanzierung waren Beitragseinnahmen in Höhe von $246 €$ je Versicherten erforderlich und somit ein um $20 \%$ höherer mittlerer Beitrag als in der PPV. Rücklagen waren zum Jahresende 2005 in der SPV mit 3 Mrd. $€$ nur in vergleichsweise geringem Umfang vorhanden.

Zwölf Jahre später hatte sich an dieser ungleichen Finanzlage grundsätzlich wenig verändert. 2017 lag der Anteil der Pflegebedürftigen in der SPV mit 4,57\% weiterhin mehr als doppelt so hoch wie in der PPV, deren Leistungsausgaben durchschnittlich nur etwas mehr als ein Viertel des entsprechenden SPVWerts betrugen. Während der Beitragssatz in der SPV stetig nach oben geklettert ist, stellt sich die Prämienentwicklung in der PPV dem- 
gegenüber vergleichsweise entspannt dar. 2017 wurden dort je Versicherten $278 €$ zur Finanzierung der jährlichen Leistungsausgaben und zur weiteren Rücklagenbildung erhoben. Dabei flossen 2017 rund $74 \%$ des Beitragsaufkommens in den Aufbau der Alterungsrückstellungen, die sich damit auf eine Gesamtsumme von 34,5 Mrd. € erhöhten (PKV 2018). Dagegen waren in der SPV 2017 durchschnittlich $500 €$ je Versicherten zur Deckung der Leistungsausgaben erforderlich.

Für einen validen Vergleich von SPV- und PPV-Versicherten müssen auch die Beihilfeausgaben berücksichtigt werden. Für aktive Beamte übernimmt die staatliche Beihilfe in der Regel $50 \%$, für Pensionäre und Angehörige $70 \%$ und für Kinder $80 \%$ der Leistungssätze der Pflegeversicherung. Knapp die Hälfte der privat Pflegeversicherten zählte 2017 zu den Beihilfeberechtigten und musste deshalb lediglich den nicht über die Beihilfe abgedeckten Teil der gesetzlichen Pflegeleistungen privat versichern. Da bundesweit keine Statistiken zu den Pflegeausgaben der Beihilfe vorliegen, werden die zusätzlichen Kosten der Beihilfestellen für die beiden Vergleichsjahre geschätzt. ${ }^{9}$ Danach sind die Leistungsausgaben je PPVVersicherten einschließlich der Beihilfeausgaben für Pflegeleistungen um rund ein Drittel höher zu veranschlagen.

\subsubsection{Auswirkungen systemübergreifender Solidarität}

Es stellt sich die Frage, wie es um die Finanzierung und Beitragsstabilität der Pflegeversicherung bestellt wäre, wenn die Politik anstelle des dualen Systems ein einheitliches Pflegeversi-

9 In der Schätzung wird von einer Verdoppelung der hälftigen Pflegeausgaben der PPV in den Jahren 2005 und 2017 ausgegangen, wenn die zusätzlichen Pflegeausgaben der staatlichen Beihilfestellen im Umfang der durch die gesetzliche Pflegeversicherung abgedeckten Leistungen berücksichtigt werden. cherungssystem nach dem Muster der SPV etabliert hätte. Empirisch lässt sich diese Frage mit einer Simulationsanalyse auf der Grundlage der SOEP-Daten beantworten, wobei sich die im Folgenden dargestellten Ergebnisse auf das Jahr 2017 beziehen. Die Simulation berücksichtigt die erwachsene Wohnbevölkerung mit insgesamt über 69 Mio. Versicherten. Dabei gilt das primäre Interesse den Beitragseinnahmen, die zu erwarten gewesen wären, wenn für alle Versicherten, also auch für die PPV-Versicherten einschließlich der beihilfeberechtigten Beamten und Pensionäre, im Analysejahr 2017 dieselben Beitragsregelungen gegolten hätten wie für die Versicherten der SPV. Das heißt speziell in Bezug auf die Beamten, dass es in der Simulation des einheitlichen Pflegeversicherungssystems keine Beihilfe gäbe, sondern wie für alle Arbeitnehmer einen hälftigen Arbeitgeberbeitrag. Datengrundlage bilden die in der Erhebungswelle 2016 des SOEP von den erwachsenen Personen abgefragten Informationen $\mathrm{zu}$ ihrem Erwerbs- und Krankenversicherungsstatus sowie zu ihren Vorjahreseinkünften. Diese wurden unter Berücksichtigung der durchschnittlichen Lohn- und Gehaltssteigerungen auf die Einkommenssituation des Jahres 2017 hochgerechnet, um hieraus die beitragspflichtigen Einnahmen abzuleiten ${ }^{10}$.

10 Die Simulationsmethode kann hier aus Platzgründen nur grob skizziert werden. In einem ersten Schritt wurden für die PPV-Versicherten Korrespondenzregeln für ihren Versichertenstatus in der einheitlichen Pflegeversicherung nach SPV-Muster festgelegt, also spezifiziert, ob eine Person als Pflichtoder als freiwilliges Mitglied, als mitversicherter Familienangehöriger, Rentner/Pensionär, ALG-I-/ALGII-Empfänger, Selbständiger oder als Student versichert wäre. Als empirische Hilfsvariablen für die Bestimmung des Versichertenstatus dienten u.a. die Informationen zum Versichertenstatus in der GKV bzw. PKV, der Erwerbsstatus und die Stellung im Beruf wie auch Informationen zum Familienstand bei PKV-Versicherten. In einem zweiten Schritt wurden die beitragspflichtigen Einkommen für jede Statusgruppe definiert und hierbei die 2017 geltenden Mindest- und Höchstbemessungsgrundlagen berücksichtigt, wie auch die pauschalen Beitragsregelungen z. B. für Studenten und ALG-II-Empfänger. Je nach Versichertenstatus wurden schließlich die 
- Tabelle 19.3 Finanzlage einer einheitlichen Pflegeversicherung nach SPV-Muster 2017 auf Grundlage der SOEP-Simulationsergebnisse für Personen 18 Jahre und älter

\begin{tabular}{|c|c|c|c|}
\hline & \multicolumn{2}{|c|}{ Zuvor versichert in: } & \multirow{2}{*}{\begin{tabular}{|l|} 
Gesamt \\
GPV*
\end{tabular}} \\
\hline & SPV & PPV & \\
\hline Anzahl Versicherte (in Tsd.) & 60.860 & 8.129 & 69.134 \\
\hline Durchschnittliche Beitragszahlungen je Versicherter in Euro p.a. & 570 & 943 & 614 \\
\hline Beitragseinnahmen in Mio. Euro & 34.667 & 7.665 & 42.395 \\
\hline Leistungsausgaben in Mio. Euro & 35.540 & 1.225 & 36.765 \\
\hline $\begin{array}{l}\text { Zusätzliche Ausgaben der Beihilfe für Beamte und Pensionäre } \\
\text { in Mio. Euro }\end{array}$ & - & 613 & - \\
\hline Leistungsausgaben inklusive Beihilfeausgaben in Mio. Euro & 35.540 & 1.838 & 37.378 \\
\hline $\begin{array}{l}\text { Saldo (Beitragseinnahmen - Leistungsausgaben) in Mio. } \\
\text { Euro }\end{array}$ & -873 & 5.827 & 5.017 \\
\hline \multicolumn{4}{|c|}{$\begin{array}{l}\text { * einschließlich Versicherte ohne Angabe zum SPV/PPV-Status } \\
\text { Quelle: Simulationsanalyse und weitere Berechnungen auf Basis SOEPv33.1; Population: Personen in Privat- } \\
\text { haushalten } 18 \text { Jahre und älter; Daten zu Leistungsausgaben: BMG 2018b und PKV } 2018 \\
\text { Pflege-Report } 2019\end{array}$} \\
\hline
\end{tabular}

Die wesentlichen Ergebnisse der Simulation sind in - Tab. 19.3 zusammengefasst. Wenn alle Pflegeversicherten 2017 in einem einheitlichen Versicherungssystem nach dem Muster der SPV versichert gewesen wären, hätte dies bei dem 2017 in der SPV erhobenen Beitragssatz von 2,55\% bzw. 2,8\% für Kinderlose zu Beitragseinnahmen von insgesamt $42,4 \mathrm{Mrd}$. $€$ geführt. Die durchschnittliche Beitragszahlung je Versicherten hätte $614 €$ im Jahr bzw. $51,16 €$ im Monat betragen. Für die Teilgruppe der SPV-Versicherten weist die Simulation Beitragseinnahmen von insgesamt $34,67 \mathrm{Mrd}$. $€$ aus, was den tatsächlichen Beitragseinnahmen der SPV im Jahr 2017 von 36,1 Mrd. € recht nahekommt. Versichertenbezogen liegt die mittlere Beitragszahlung je SPV-Versicherten bei $570 € \mathrm{im} \mathrm{Jahr} \mathrm{bzw.} \mathrm{47,50€} \mathrm{im} \mathrm{Monat.}$

Für die rund 8,1 Mio. PPV-Versicherten wären nach den Beitragsregelungen der SPV im Jahr 2017 insgesamt Beiträge in Höhe von rund

Beiträge auf Grundlage des 2017 geltenden SPVBeitragssatzes von 2,55 bzw. 2,8 \% für Kinderlose für das Gesamtjahr berechnet.
7,67 Mrd. € gezahlt worden bzw. je Versicherten von durchschnittlich $943 € \mathrm{im} \mathrm{Jahr} \mathrm{bzw.} \mathrm{78,58€}$ im Monat. Dies übersteigt die 2017 tatsächlich an die PPV geleisteten Prämienzahlungen von rund 2,6 Mrd. € beträchtlich. Dafür gibt es zwei Gründe: Zum einen würden auch die bislang von der staatlichen Beihilfe geleisteten Pflegeaufwendungen von geschätzt $0,61 \mathrm{Mrd} . €$ in dem simulierten einheitlichen Versicherungssystem über Beiträge finanziert. Zum anderen und vor allem würden die PPV-Versicherten entsprechend ihrer Einkommenslage an der solidarischen Finanzierung der Pflegeversicherung beteiligt. Wer etwa 2017 über beitragspflichtige Einnahmen oberhalb der Beitragsbemessungsgrenze von monatlich $4.350 €$ verfügte, hätte - einschließlich des Arbeitgeberbeitrags - ebenso den Höchstbeitrag von knapp $111 €$ bzw. für Kinderlose von $121,80 €$ entrichten müssen wie alle SPV-Versicherten mit gleich hohem Einkommen auch.

Der Nettoeffekt einer Einbeziehung der PPV-Versicherten in ein einheitliches Pflegeversicherungssystem nach dem Muster der SPV hätte 2017 rund 5,8 Mrd. € betragen, resultie- 
rend aus dem Saldo zwischen den simulierten Beitragseinnahmen und den Leistungsausgaben der zuvor PPV-Versicherten sowie den bislang von den Beihilfekassen von Bund und Ländern getragenen Pflegeleistungen für Beamte und Versorgungsempfänger. Dieser Betrag entspricht rund 0,4 Beitragssatzpunkten. Dieser rechnerische Entlastungseffekt ist damit etwas höher als in einer Simulationsanalyse auf der Grundlage der Einkommens- und Verbrauchsstichprobe 2013 (Rothgang und Domhoff 2017).

\subsection{Fazit}

Rund ein Vierteljahrhundert nach ihrer Einführung steht die Finanzierung der gesetzlichen Pflegeversicherung erneut auf dem Prüfstand. In diesem Beitrag sind zwei wesentliche Anknüpfungspunkte für entsprechende Strukturreformen betrachtet worden: die Einführung eines steuerfinanzierten Bundeszuschusses sowie die in hohem Maße unausgewogene Lastenverteilung zwischen den beiden Versicherungszweigen der SPV und PPV.

Im Ergebnis ist die Finanzierungsoption Bundeszuschuss in diesem Beitrag deutlich skeptischer beurteilt worden, als dies in der aktuellen Debatte oftmals der Fall ist. Das betrifft zunächst die Frage, inwieweit die SPV gesamtgesellschaftliche Aufgaben („versicherungsfremde Leistungen“) erfüllt, deren Ausgaben sachadäquat durch die Steuerzahler zu finanzieren wären. Dies gilt aber erst recht jenseits dieses Sachzusammenhangs. Wenn es in dem bereits zu Beginn zitierten Entschließungsantrag von Hamburg und drei weiteren Bundesländern heißt, dass sich ein steuerfinanzierter Bundeszuschuss zu SPV „in einem ersten Schritt“ am Umfang der gesamtgesellschaftlichen Aufgaben orientieren sollte (Bundesrat 2019), stellt sich automatisch die Frage nach der Begründung für einen möglichen zweiten Schritt und dessen konzeptionelle Ausgestaltung.

Eine andere Frage ist die Herstellung systemübergreifender Solidarität in der gesamten gesetzlichen Pflegeversicherung - etwa im Rahmen einer Pflegebürgerversicherung. Die hier vorgestellte Simulation hatte nicht das Ziel, sämtliche Finanzwirkungen des Einbezugs aller Pflegeversicherten in die solidarische Pflegefinanzierung nach dem Muster der SPV abzuschätzen. Speziell die längerfristige Entlastung der Beihilfesysteme von Bund und Ländern bedürfte einer weiteren eigenständigen Analyse. Hier ging es in erster Linie darum, die oben zitierte Prämisse des Bundesverfassungsgerichts für die Einführung der „Pflegevolksversicherung “ in Gestalt von zwei Versicherungszweigen empirisch $\mathrm{zu}$ überprüfen, insofern also die Ausgewogenheit der Lastenverteilung zu beurteilen. Wie die Ergebnisse belegen, kann von einer ausgewogenen Lastenverteilung nicht die Rede sein. Die SPVVersicherten tragen ein deutlich höheres Pflegerisiko als die PPV-Versicherten, die ihrerseits im Durchschnitt über ein mehr als doppelt so hohes Bruttogesamteinkommen verfügen. Im Ergebnis dieser gravierenden Strukturunterschiede liegt der absolute Durchschnittsbeitrag, den die SPV-Versicherten zu entrichten haben, weit über der Durchschnittsprämie der im Mittel deutlich besserverdienenden PPVVersicherten, obwohl aus deren Beitragszahlungen nicht nur die laufenden Leistungsausgaben bestritten, sondern zusätzlich noch Alterungsrückstellungen gebildet werden.

Die vom Bundesverfassungsgericht als Voraussetzung für die Dualität von SPV und PPV postulierte Ausgewogenheit in der Lastenverteilung ist damit seit Einführung der Pflegeversicherung in erheblichem Umfang verletzt worden. Hiervor im Hinblick auf nachhaltig wirksame Finanzierungsreformen die Augen $\mathrm{zu}$ verschließen und die bestehenden Privilegien der PPV-Versicherten unverändert fortzuschreiben könnte letztlich nicht nur das Prinzip der solidarischen Finanzierung in der SPV gefährden, sondern darüber hinausgehend den vielbeschworenen Zusammenhalt der gesamten Gesellschaft. Von der Sache her ist es unverständlich, warum dieses Thema nicht längst wieder auf der politischen Agenda steht, nachdem bereits im Koalitionsvertrag der ersten 
„GroKo“ von 2005 - allerdings folgenlos - angekündigt worden war, dass „zum Ausgleich der unterschiedlichen Risikostrukturen“ ein Finanzausgleich zwischen SPV und PPV eingeführt werden sollte (CDU/CSU/SPD 2005, S. $91 \mathrm{f}$.).

Ergebnis einer ausgewogeneren Lastenverteilung - ob nun durch die Einführung einer Pflegebürgerversicherung (Deutscher Bundestag 2019) oder einen Finanzausgleich zwischen SPV und PPV - wäre eine spürbare finanzielle Entlastung in der sozialen Pflegeversicherung. Die vorgestellte Simulation zeigt allerdings auch, dass selbst bei einer sofortigen Einführung einer Pflegebürgerversicherung was verfassungsrechtlich keineswegs risikolos ist - der Entlastungseffekt begrenzt ist. Wie sich der Transfer mit der fortschreitenden Alterung auch in der PPV weiterentwickelt, ist - unter Berücksichtigung der bestehenden Strukturunterschiede und Selektionseffekte - noch nicht abzusehen. Nichtsdestoweniger könnten die zusätzlichen Mittel kurz- bis mittelfristig eine wichtige Finanzierungsquelle für eine verbesserte Bezahlung von Pflegekräften und die Einstellung neuen Personals darstellen.

\section{Literatur}

ASMK (2018) Externes Ergebnisprotokoll der 95. Konferenz der Minister und Ministerinnen, Senatoren und Senatorinnen für Arbeit und Soziales der Länder. https://asmkintern.rlp.de/de/beschluesse/. Zugegriffen: 1. Apr. 2019

BMG; Bundesministerium für Gesundheit (2018a) Kabinett beschließt Anhebung des Pflegebeitrags. Bundesgesundheitsminister Spahn: Gute Pflege muss uns als Solidargemeinschaft etwas wert sein (Pressemitteilung Nr. 21 vom 10.10.2018)

BMG; Bundesministerium für Gesundheit (2018b) Zahlen und Fakten zur Pflegeversicherung. Stand 11.07.2018

Bundesrat (2019) Entschließung des Bundesrates zur Weiterentwicklung der Pflegeversicherung. Antrag der Länder Hamburg, Berlin, Bremen, SchleswigHolstein. Drucksache 106/19 vom 01.03.2019

BVerfG; Bundesverfassungsgericht (2001a) Urteil des Ersten Senats vom 3. April 2001 - 1 BvR 1629/94

BVerfG; Bundesverfassungsgericht (2001b) Urteil des Ersten Senats vom 3. April 2001 - 1 BvR 2014/95
CDU, CSU, SPD (2005) Gemeinsam für Deutschland - mit Mut und Menschlichkeit. Koalitionsvertrag zwischen CDU, CSU und SPD vom 11.11.2005

DAV; Deutsche Aktuarvereinigung (2015) Herleitung der Rechnungsgrundlagen DAV 2008 P für die Pflegerenten(zusatz)versicherung. Richtlinie. Köln, 15.06.2015. https://aktuar.de/unsere-themen/ lebensversicherung/sterbetafeln/UT_LV_16.pdf. Zugegriffen: 1. Apr. 2019

Deutscher Bundestag (2019) Pflege gerecht und stabil finanzieren - Die Pflege-Bürgerversicherung vollenden. Drucksache 19/8561 vom 20.03.2019 (Antrag der Fraktion BÜNDNIS 90/DIE GRÜNEN. Berlin)

Dräther H, Jacobs K, Rothgang H (2009) PflegeBürgerversicherung. In: Dräther $H$, Jacobs $K$, Rothgang $\mathrm{H}$ (Hrsg) Fokus Pflegeversicherung. Nach der Reform ist vor der Reform. KomPart, Berlin, S 7192

GKV-Spitzenverband (2013) Faktenblatt. Thema: Ausgaben für versicherungsfremde Leistungen (Pressestelle GKV-SV, 04.03.2013)

GKV-Spitzenverband (2018a) Stellungnahme des GKVSpitzenverbandes vom 22.11.2018 zum Entwurf eines Fünften Gesetzes zur Änderung des Elften Buches Sozialgesetzbuch - Beitragssatzanpassung. (Drucksache 19/5464) vom 06.11. 2018. Deutscher Bundestag. GKV-Spitzenverband, Berlin (Ausschussdrucksache 19(14)0047(8.2).)

GKV-Spitzenverband (2018b) Kennzahlen der sozialen Pflegeversicherung. https://www.gkvspitzenverband.de/media/grafiken/pflege_ kennzahlen/spv_kennzahlen_03_2018/SPV_ Kennzahlen_Booklet_03-2018_300dpi_2018-0315.pdf. Zugegriffen: 12. Dez. 2018

Goebel J, Grabka M, Liebig S, Kroh M, Richter D, Schröder C, Schupp J (2018) The German Socio-Economic Panel (SOEP). Jahrb Natl Okon Stat. https://doi.org/10. 1515/jbnst-2018\T1\textendash0022

Greß S, Bieback H-J (2014) Steuerfinanzierung in der Gesetzlichen Krankenversicherung - keine Verlässlichkeit und Stetigkeit. ifo Schnelldienst 67(7):6-9

Haun D (2013) Quo vadis, GKV und PKV? Entwicklung der Erwerbs- und Einkommensstrukturen von Versicherten im dualen System. In: Jacobs K, Schulze S (Hrsg) Die Krankenversicherung der Zukunft. Anforderungen an ein leistungsfähiges System. KomPart, Berlin, S 75-105

PKV; Verband der privaten Krankenversicherung (2006) Zahlenbericht der privaten Krankenversicherung 2005/06. PKV, Köln

PKV (2017) Zahlenbericht der privaten Krankenversicherung 2016. PKV, Köln

PKV (2018) Zahlenbericht der privaten Krankenversicherung 2017. PKV, Köln

Rothgang H (2018) Stellungnahme zum Antrag der Fraktion DIE LINKE „Eigenanteile in Pflegeheimen senken - Menschen mit Pflegebedarf finanziell ent- 
lasten“. BT-Drucksache 19/960 anlässlich der öffentlichen Anhörung des Ausschusses für Gesundheit des Deutschen Bundestages am 4. Juni 2018. Deutscher Bundestag, Berlin (Ausschussdrucksache 19(14)0014(15) vom 04.06.2018)

Rothgang H, Domhoff D (2017) Beitragssatzeffekte und Verteilungswirkungen der Einführung einer „Solidarischen Gesundheits- und Pflegeversicherung". Gutachten im Auftrag der Bundestagsfraktion DIE LINKE und der Rosa-Luxemburg-Stiftung, Bremen. https://www.rosalux.de/publikation/id/37468/ solidarische-gesundheits-und-pflegeversicherung/. Zugegriffen: 1. Apr. 2019
Schwinger A, Rothgang H, Kalwitzki T (2018) „Die Pflegeversicherung boomt": Mehrausgaben der Pflegeversicherung - Retrospektive und Projektion. Gesundh Sozialpolit 72(6):13-22

Spahn J (2018) „Wer vor Verantwortung wegläuft, verliert". Gesundheitsminister Jens Spahn über den Zustand der Koalition, die Pflichten der Regierung und seine Pläne für die Pflege. Der Tagesspiegel vom 16.10.2018, S. 6

Statistisches Bundesamt (2018) Pflegestatistik 2017. Pflege im Rahmen der Pflegeversicherung. DeStatis, Wiesbaden

Open Access Dieses Kapitel wird unter der Creative Commons Namensnennung 4.0 International Lizenz (http:// creativecommons.org/licenses/by/4.0/deed.de) veröffentlicht, welche die Nutzung, Vervielfältigung, Bearbeitung, Verbreitung und Wiedergabe in jeglichem Medium und Format erlaubt, sofern Sie den/die ursprünglichen Autor(en) und die Quelle ordnungsgemäß nennen, einen Link zur Creative Commons Lizenz beifügen und angeben, ob Änderungen vorgenommen wurden.

Die in diesem Kapitel enthaltenen Bilder und sonstiges Drittmaterial unterliegen ebenfalls der genannten Creative Commons Lizenz, sofern sich aus der Abbildungslegende nichts anderes ergibt. Sofern das betreffende Material nicht unter der genannten Creative Commons Lizenz steht und die betreffende Handlung nicht nach gesetzlichen Vorschriften erlaubt ist, ist für die oben aufgeführten Weiterverwendungen des Materials die Einwilligung des jeweiligen Rechteinhabers einzuholen. 